\title{
The ATCA/VLA OH 1612 MHz survey
}

\section{Observations of the galactic Disk region ${ }^{\star}$}

\author{
M.N. Sevenster ${ }^{1}$, J.M. Chapman ${ }^{2,3}$, H.J. Habing ${ }^{1}$, N.E.B. Killeen ${ }^{3}$, and M. Lindqvist ${ }^{4}$ \\ 1 Sterrewacht Leiden, P.O. Box 9513, 2300 RA Leiden, The Netherlands \\ 2 Anglo Australian Observatory, P.O. Box 296, Epping 2121 NSW, Australia \\ 3 Australia Telescope National Facility, P.O. Box 76, Epping 2121 NSW, Australia \\ 4 Onsala Space Observatory, S-439 92 Onsala, Sweden
}

Received October 22, accepted December 10, 1996

\begin{abstract}
We present observations of the region between $-45^{\circ} \leq \ell \leq-10^{\circ}$ and $|b| \leq 3^{\circ}$ in the $\mathrm{OH} 1612.231 \mathrm{MHz}$ line, taken in 1994 January with the Australia Telescope Compact Array (ATCA) ${ }^{1}$. These observations are part of a larger survey, covering $|\ell| \leq 45^{\circ}$ and $|b| \leq 3^{\circ}$, with the ATCA and the Very Large Array. In a previous article we have discussed the observational strategy in detail. In this paper we present only the objects found in this part of the survey. The region was systematically searched for $\mathrm{OH}$-maser emission and was covered completely with 910 pointing centres separated by $30^{\prime}$. We found $202 \mathrm{OH}-$ masing objects, 112 of which are new detections. Out of the 202 objects, 166 have a standard double-peaked spectral profile, 32 a single-peaked profile and 4 objects have non-standard or irregular profiles.

In this article we analyse the data statistically and give classifications and identifications with known sources where possible. The astrophysical, kinematical, morphological and dynamical properties of subsets of the data will be addressed in future articles ${ }^{2}$.
\end{abstract}

Key words: surveys - stars: AGB and post-AGB Galaxy: stellar content — radio lines: stars

Send offprint requests to: M.N. Sevenster

* Supplement Series

1 The Australia Telescope Compact Array is operated by the Australia Telescope National Facility, CSIRO, as a national facility.

2 The electronic version of this paper, including Table 1 and the spectra of Fig. 6, can be obtained from http://www.ed-phys.fr. Table 1 is available via anonymous ftp (130.79.128.5) or through the World Wide Web (http://cdsweb.u-strasbg.fr/Abstract.html).

\section{Introduction}

We have surveyed a large area of the galactic plane in the $\mathrm{OH} 1612 \mathrm{MHz}$ satellite line. The aim of the survey was to sample the stellar dynamics in the plane, cutting through the major components of the Galaxy. Very strong radiation is emitted in the observed $\mathrm{OH}$ line by, amongst others, OH/IR stars (for a review see Habing 1996). These are particularly suitable tracers of the stellar dynamics for a variety of reasons. Most important is that they are easily observable in any region of the Galaxy, their line-of-sight velocity can be determined very accurately and they represent a large range of the stellar population. This article is the sequel of a previous article (Sevenster et al. 1996, Paper I), that we refer to for a detailed description of the survey, the objects and the reductional strategy. In Paper I we discussed the region between $|\ell| \leq 10^{\circ}$ and $|b| \leq 3^{\circ}$, the "Bulge region". Here we discuss the results of the connecting region, between $-45^{\circ} \leq \ell \leq-10^{\circ}$ and $|b| \leq 3^{\circ}$, the "Disk region". We will not give details of the observations, reduction and analysis, but mention only the most important parameters and those that differ significantly for the two regions of the survey, such as precise completeness levels and velocity coverage. We will discuss the observations briefly in Sect. 2 and the data reduction in Sect. 3. In Sect. 4 we give the results of the survey in the Disk region and in Sect. 5 a statistical analysis of the data, along the same lines as that in Paper I.

\section{Observations}

The observations were taken with the Australia Telescope Compact Array in 1994 January and September in 6Aconfiguration. The resolution is $6^{\prime \prime}$ in right ascension and $\sim 10^{\prime \prime}$ in declination. The full width at half maximum (FWHM) of the primary beam (PB) of the antennae at the frequency of the observations is 29.7. The area of the Disk region was covered with 910 pointing 

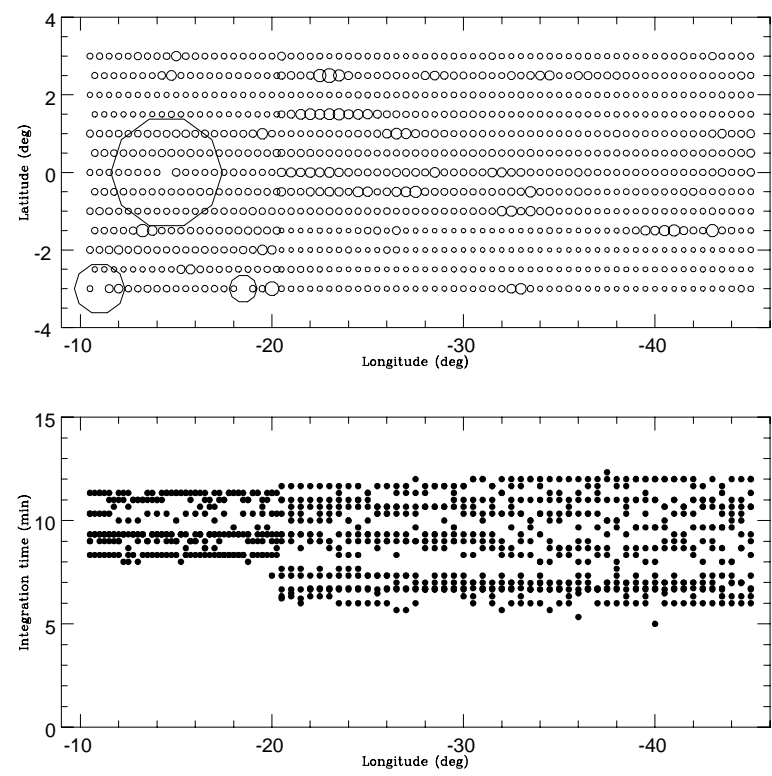

Fig. 1. Upper panel: noise levels as function of pointing centre, with the diameter of the circles proportional to the noise in the field. Lower panel: used integration times as function of longitude (the scatter is for the different latitudes)

centres, separated by $30^{\prime}$. The coverage is complete for offset 21.2 which translates to a primary-beam response (PBR) of 0.25. For observational convenience, the observations were taken in two "blocks"; one for $\ell>-20^{\circ}$ (part B) and one for $\ell \leq-20^{\circ}$ (part A) as can be seen in Fig. 1. In part $\mathrm{A}$ the pointing centres are not distributed in the honeycomb pattern described in Paper I, but rather in a rectangular pattern, for reasons of temporary observational incompetence. The data were taken in two linear polarizations, using a total bandwidth of $4 \mathrm{MHz}$ and 1024 spectral channels (channel separation $3.9 \mathrm{kHz}$, correlator frequency resolution $4.69 \mathrm{kHz}$ ). The spectral band was centred at $1612 \mathrm{MHz}$, offset by $0.231 \mathrm{MHz}$ from the rest frequency of the $\mathrm{OH}$ transition. No Doppler tracking was used during the observations. The velocity coverage is $\left(-295,+413 \mathrm{~km} \mathrm{~s}^{-1}\right)$ for all fields except those with $b=0^{\circ}$ in part A, for which the coverage is $\left(-300,+379 \mathrm{~km} \mathrm{~s}^{-1}\right)$ because they were partly observed in September. The data were Hanning smoothed to give a velocity resolution of $1.46 \mathrm{~km} \mathrm{~s}^{-1}(7.8 \mathrm{kHz})$. The observations were taken using a "mosaic" mode, to optimize uv-sampling (for details see Paper I). The secondary calibrators observed in the Disk region were 1740-517 (part B) and 1414-590 (part A). The primary calibrators are the same as in the Bulge region, 1934-638 and/or 0823-500. The average integration time per pointing is 9 min, resulting in an average noise level in empty cubes of $29.6 \mathrm{mJy}$.

\section{Data reduction}

The data were reduced as described in Paper I, using the reduction package Miriad (Sault et al. 1995). The data were searched with an adapted version of the CLEAN algorithm, fully described in Appendix A of Paper I. This was performed on a Cray-C98. During the observations for the Disk region, especially its parts furthest away from the galactic Centre, considerable narrow-band radio-frequency interference (RFI) was present. To minimize this, we performed two steps of removal of RFI. Before any searching of the data we automatically traced and flagged any time-cuts that contained RFI spikes. After removal of the brightest sources, we fitted $11^{\text {th }}$-order polynomials (UVLIN routine in Miriad, Sault 1994, see Paper I) to all visibilities in order to remove wide-band RFI. In part B of the Disk region hardly any narrow-band RFI was found present, however, in part A severe narrowband RFI was present in $\sim 20 \%$ of the fields. After flagging the time-cuts with RFI, the serviceable integration time on these fields was reduced on average by $\sim 30 \%$ (see Fig. 1). Still, even in the fields with shortest integration times, the integration times are similar to those in the Bulge region (Paper I). The source-finding algorithm is as described in the appendix of Paper I. The lowest level of searching was performed with minimum flux density of $90 \mathrm{mJy}$. The cell sizes used are $5^{\prime \prime} \times 10^{\prime \prime}$ in the first two passes of source finding and $2.5 \times 5^{\prime \prime}$ in subsequent passes.

\section{Results}

In Table 1 all narrow-line $\mathrm{OH}$ sources found are listed. In total there are 202 sources, 90 of which have been identified with known $\mathrm{OH}$-satellite-line masers. The references for previous $\mathrm{OH}$ detections are given in Table 2. We visually identified 166 double-peaked (D), 32 single-peaked (S) and 4 irregular (I) sources. A reliable IRAS identification is found for 151 sources.

For each source the table gives an entry number (Col. 1), the $\mathrm{OH} \ell-b$ name (Col. 2), a type (D, S, I) identifier (Col. 3), position in J2000 coordinates (Cols. 4, 5), a measure of the error in the positions (Col. 6), the distance from the source to the pointing centre (Col. 7), the peak, stellar and outflow velocities (Cols. 8 to 11), the peak fluxes (Cols. 12, 13), the noise in the field where the source was detected (Col. 14, velocity resolution $1.46 \mathrm{~km} \mathrm{~s}^{-1}$ ), the number of the reference to previous observations if applicable (Col. 15), the name of the nearest IRAS point source (Col. 16) and the distance to this nearest IRAS point source expressed as a fraction of the corresponding IRAS error ellipse (Col. 17). Table 1 can be retrieved from the WWW (http://cdsweb.u-strasbg.fr/Abstract.html).

In Fig. 2 the longitude-latitude diagram and longitudevelocity diagramm are shown for all 202 sources. The spectra for all sources are shown in Fig. 6. They are 

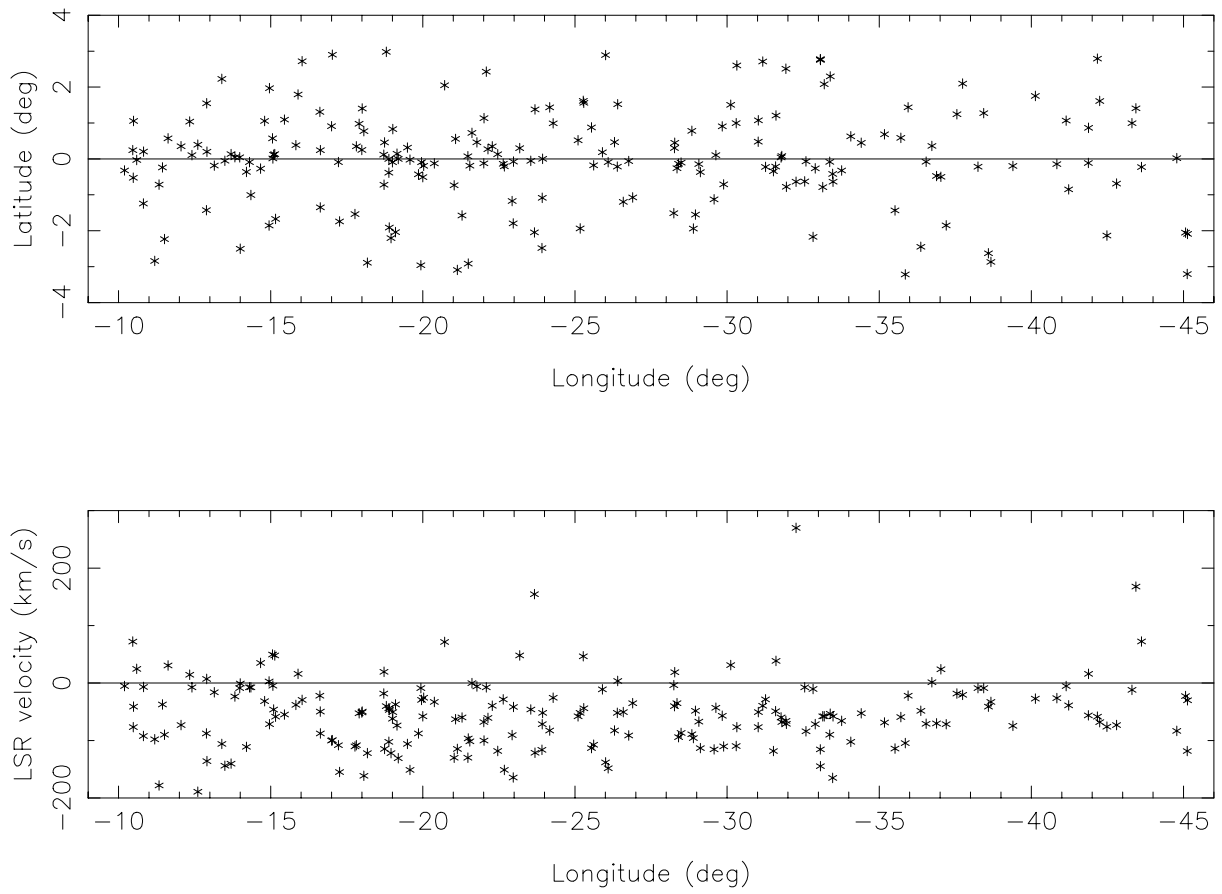

Fig. 2. The longitude-latitude and longitude--velocity distributions for all the sources in the galactic Disk region
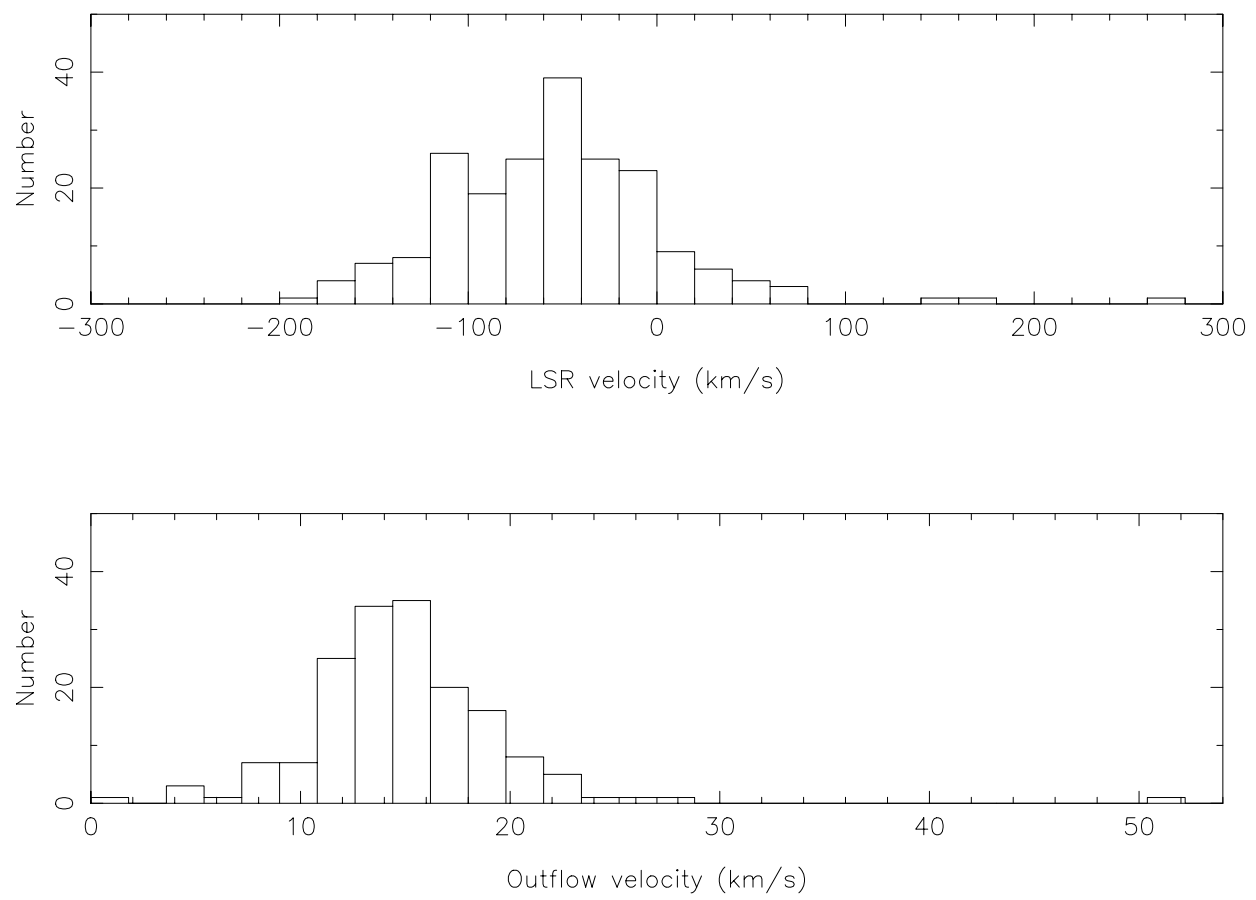

Fig. 3. The histograms of the line-of-sight velocity and the expansion velocity of all the sources in the galactic Disk region. The mean expansion velocity is $15 \mathrm{~km} \mathrm{~s}^{-1}$ 
displayed with $50 \mathrm{~km} \mathrm{~s}^{-1}$ on either side of the stellar velocity,

except for \#71 that has an outflow velocity higher than $50 \mathrm{~km} \mathrm{~s}^{-1}$. The peaks that were detected at the position of the object are marked by dots on top. For source \#46 that has a very irregular spectrum, we marked the whole range of detected velocities for the broad peak. The stellar velocities of the sources in this region of the Galaxy have a much smaller spread than those of the sources in the Bulge region (Fig. 3). Therefore, the sidelobe problem (Paper I) is even more prominent. Spectrum \#167 shows a particularly clear example of this, where the real peak of the source is almost masked out by a sidelobe from a neighbouring star that has a higher flux density at this position than even the source itself. Another example to be noted is spectrum \#55 that has a strong negative sidelobe from source \#56. For details on the extraction of the spectra see Paper I.

\section{Data analysis}

In this section we analyse the global completeness of the survey and discuss the statistical accuracy of the paremeters given in Table 1 . We will treat each parameter only briefly and refer to Paper I for the details. We will assume errors are normally distributed, unless stated otherwise.

\subsection{Survey completeness}

\subsubsection{Noise levels}

The empirical noise in the empty cubes in the Disk region, especially part $\mathrm{A}$, is more variable than in the Bulge region, because RFI was more prominent (see Sect. 3). In Fig. 1 we show the noise levels for each pointing. The average noise is $29.6 \mathrm{mJy}$; $90 \%$ of the fields have noise levels below $35 \mathrm{mJy}$. There are three fields with noise levels higher than explicable by high system temperatures or short integration times alone, where the higher noise was due to temporary correlator errors.

\subsubsection{Detection levels}

In this section we treat the global completeness of the sample. The absolute detection level in the searching routine was set to $90 \mathrm{mJy}$ (cf. $120 \mathrm{mJy}$ in Bulge region), corresponding to three times the average noise level. Figure $4 \mathrm{a}$ shows the primary beam response $(\mathrm{PBR})$ at $18 \mathrm{~cm}$ as a function of offset (Wieringa \& Kesteven 1992). For inner fields, the largest possible offset for a source is $21^{\prime}$ (cf. $19^{\prime}$ for the Bulge region), which coincides with the half width at 0.25 of the global maximum.

Figure 4b shows the PBR (from Fig. 4a) of each source against its peak flux density. The solid line connects stars with the lowest detected $\mathrm{OH}$ flux densities, determined in PBR bins of width 0.1 . The dashed line in Fig. $4 \mathrm{~b}$ indicates the expected relation between PBR and flux density cut-off for an absolute detection limit of $140 \mathrm{mJy}$, which is the best fit to the extremes in the solid line. As in Paper I, we find that this is 4 times the typical noise of $35 \mathrm{mJy}$ (cf. $40 \mathrm{mJy}$ Bulge region). This $4 \sigma$ cut-off is a global statistic, i.e. in terms of the average noise and global minimum flux density. Locally, i.e. taking the noise and the minimum flux density per field, the cut-off is at $6 \sigma$ as can be seen in Fig. 4f, where we plotted the SNR for all objects with SNR $<40$ against their offset. This does not alter the validity of using $140 \mathrm{mJy}$ as the globally limiting, observed flux density ${ }^{3}$. This part of the survey is therefore theoretically complete for flux densities above $140 \mathrm{mJy} / 0.25$ is $560 \mathrm{mJy}$ (cf. $500 \mathrm{mJy}$ for the Bulge region).

In Fig. 4c we plot the cumulative flux density distributions for the detected sources with $\mathrm{PBR}>0.8$ (solid line) and, for comparison, with $\mathrm{PBR}<0.6$ (dashed line). We estimate that the survey was essentially complete for $\mathrm{PBR}>0.8$ and take the solid line in Fig. 4c as an approximation to the intrinsic cumulative flux density distribution for the $\mathrm{OH} / \mathrm{IR}$ stars in the present sample. From this distribution, together with the information from Figs. 4a,b, we derive the relative completeness of the survey as a function of offset, plotted in Fig. $4 \mathrm{~d}$. Using the absolute cut-off of $140 \mathrm{mJy}$ (Fig. 4b), we integrate the relation shown in Fig. $4 \mathrm{~d}$ from the largest offset possible for a source with a certain flux density inward to offset $0^{\prime}$, normalized with the exact area of the $\mathrm{PB}$ at a certain offset. This yields the completeness of the survey as a function offlux density (Fig. 4e). From Fig. 4e, we find an empirical completeness of $99 \%$ at $500 \mathrm{mJy}$ and $80 \%$ for sources brighter than $275 \mathrm{mJy}$ (cf. $300 \mathrm{mJy}$ for the Bulge). The curve of completeness versus flux density does not rise as steeply as that for the Bulge sources.

Summarizing, this data set is $99 \%$ complete for flux densities higher than $500 \mathrm{mJy}$ and has an absolute cut-off at $140 \mathrm{mJy}$. The completeness levels are very close to those in the Bulge region, with the main differences in low flux densities at small (!) offsets, despite different observational parameters.

\subsection{Positions}

The treatment of the positional errors is the same as described in Paper I and we refer to it for details (see in particular its Fig. 5). The errors are of the order of 0.5 , with the biggest contribution in the declination. The entry $\Delta$ in Table 1, Col. 6 gives a high upper limit to the positional errors (see Paper I). The average value of $\Delta$ in Table 1 is $0 . \prime 65$. The sources with highest values of $\Delta$ are in general the brightest ones.

\footnotetext{
3 The reason for this apparent controversy is that the average surface density in this sample is only 0.9 stars per square degree (cf. 2.4 in the Bulge). Therefore, the statistical fluctuations are larger and there were simply no faint stars observed in the inner regions of the $\mathrm{PB}$.
} 

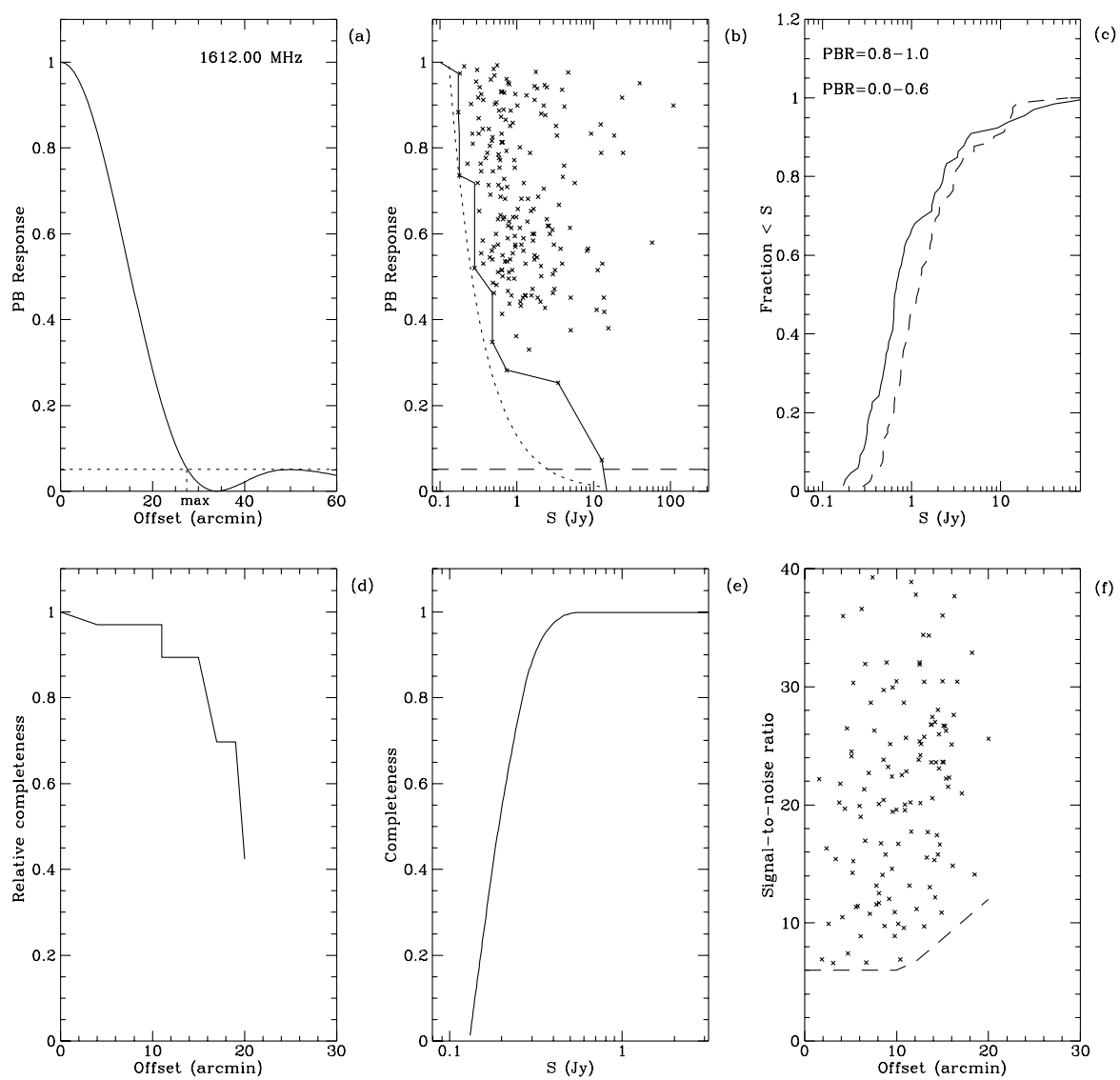

Fig. 4. Representation of the completeness of the data. a) The primary beam response (PBR) of the ATCA antennae at 18 $\mathrm{cm}$, as a function of radial offset from the pointing centre, taken from Wieringa \& Kesteven (1992). The offset labelled "max" indicates the offset to which the measured flux density of a genuine source is always higher than the flux density measured for a "ghost" image of that source. b) The PBR calculated for the detected sources plotted against the highest peak-OH flux densities, corrected for primary beam attenuation. The solid line indicates the lowest flux densities detected for PBR bins of width 0.1. The dashed line indicates the expected inner boundary calculated from the PBR curve in (a) for a limiting flux density of $140 \mathrm{mJy}$ (chosen to fit observed extremes). c) The cumulative flux density distribution for stars with PBR values $>$ 0.8 (solid line) and $<0.6$ (dashed line). The solid line is taken to be the intrinsic $\mathrm{OH}$ flux density distribution for the sources in the survey. d) The completeness, relative to the pointing centres, of the survey as a function of position offsets from the field centres. An offset of $15^{\prime}$ corresponds to half the distance between nearest fields in a row of constant latitude (Fig. 1); 26 '.5 is the largest observed offset in the survey. e) The completeness of the sample as a function of flux density. The offset out to which a source with certain flux density can be observed is determined from the dotted line in (b). Then we integrate the completeness given in (d) from that offset to zero, normalizing with the surface of the PB. f) The SNR for all sources with SNR lower than 40, plotted against their radial offset from the pointing centre. The dashed line shows the (observed) lower limit for the SNR at a certain offset

\subsection{Flux densities}

The flux densities given in Table 1 are measured in the channel of the highest peak. They are necessarily valid only for the velocity resolution of $1.46 \mathrm{~km} \mathrm{~s}^{-1}$. The treatment of the errors in the flux densities is described in detail in Paper I. There is a systematic error of about $-5 \%$, arising from interpolation of the frequency channels into velocity channels, and an additional random error of about $5 \%$.

\subsection{Velocities}

The typical error for all velocities given in Table 1 (Cols. $8-11$ ) is $1 \mathrm{~km} \mathrm{~s}^{-1}$ (see Paper I). The velocity coverage of this part of the survey was virtually constant (see Sect. 2). Therefore the band-edge effects discussed in Paper I are 
of no importance here, all the more because no detections were made at velocities lower than $-200 \mathrm{~km} \mathrm{~s}^{-1}$ or higher than $270 \mathrm{~km} \mathrm{~s}^{-1}$. Since we are sampling the Disk of the Galaxy in this region of the sky the velocities are much more confined to a narrow range due to regular rotation (Fig. 3).

\subsection{IRAS identifications}

In Col. 16 the nearest point source (PS) from the IRAS point-source catalogue (PSC) is given, together with, in Col. 17, the distance from the $\mathrm{OH}$ position in Cols. 3, 4 to the position of that IRAS PS expressed in fractional radii of the IRAS error ellipse in the direction of the $\mathrm{OH}$ position (see Paper I). Of the 202 sources, 151 (75\%) have an IRAS PS counterpart within the IRAS error ellipse $(N<1)$ and the average distance for all sources from $\mathrm{OH}$ position to IRAS position is 30 '. 4 . The fraction of sources with good IRAS identification is slightly higher and the average distance from $\mathrm{OH}$ - to IRAS position is slightly smaller than for the sources in Paper I. Figure 5 shows the IRAS two-colour diagram for sources with an IRAS identification, that lies within the IRAS error ellipse and has reliable IRAS colours. Features in this diagram will be discussed in future articles. However, we note that the Disk sources are much more confined to the so-called evolutionary track as defined by van der Veen \& Habing (1988).

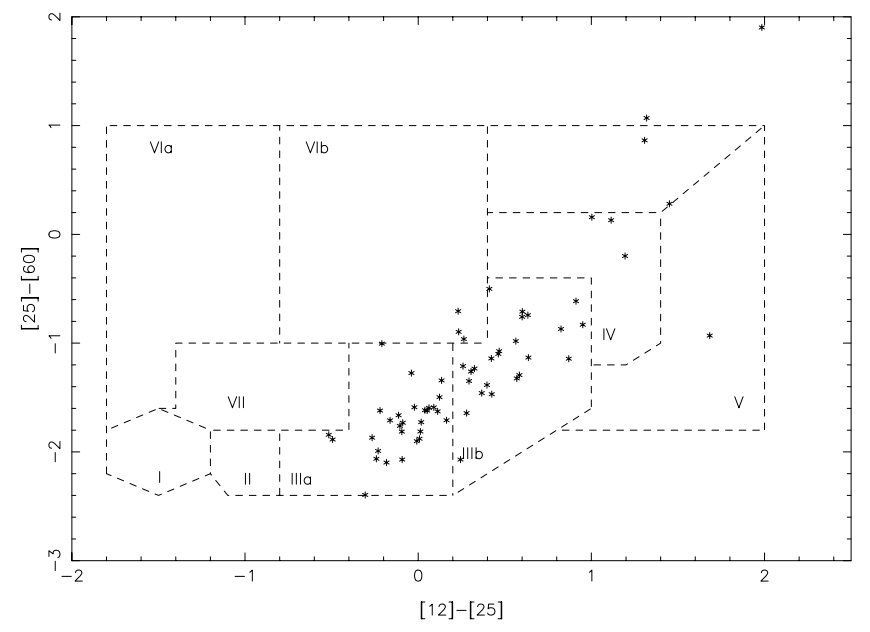

Fig. 5. The IRAS two-colour diagram for sources with an IRAS identification lying within the IRAS error ellipse (Col. 17, $N \leq 1$ ) with well-determined IRAS 12, 25 and $60 \mu \mathrm{m}$ flux densities (i.e. no upper limits). The colours are defined as [12] $-[25]=2.5^{10} \log \left(S_{25} / S_{12}\right)$.

\section{6. $O H$ identifications}

The references given in Col. 15 are to previously known $\mathrm{OH} 1612 \mathrm{MHz}$ maser positions. They were determined with the SIMBAD database, searching for sources with $\mathrm{OH}-$ catalogue names within one arcminute from the position in Col. 3, 4. The same conditions mentioned in Paper I apply; there is no "fool-proof" method of identification. Out of the 202 sources, 90 are found to have such an $\mathrm{OH}$ identification. Source \#190 is known to be a planetary nebula (NGC 6302) and has been detected in $\mathrm{OH}$ bij Zijlstra et al. (1989) but does not appear with an $\mathrm{OH}$ name in standard catalogues and therefore does not have an entry in Col. 15. Sources \#198 and \#199 are re-detections from the Bulge region.

\section{Summary}

We have given the results of a survey of the region $-45^{\circ} \leq$ $\ell \leq-10^{\circ}$ and $|b| \leq 3^{\circ}$ in the $\mathrm{OH} 1612.231 \mathrm{MHz}$ maser line. The survey is $99 \%$ complete for sources brighter than $500 \mathrm{mJy}$ and $80 \%$ complete for sources brighter than $275 \mathrm{mJy}$. The absolute flux density limit is $140 \mathrm{mJy}$. We have found 202 compact $\mathrm{OH}$-maser sources, 112 of which are new detections. The sources are mainly $\mathrm{OH} / \mathrm{IR}$ stars, with a few related sources, like planetary nebulae. The sources have positions accurate to $0 . .5$, velocities accurate to $1 \mathrm{~km} \mathrm{~s}^{-1}$ and flux densities accurate to 5\%. For 151 sources, an associated IRAS point source is found. All the statistical properties of this dataset, most importantly the completeness levels, are similar enough to the Bulge data set, for the two regions to be treated statistically as a single data set.

Acknowledgements. MS thanks the NFRA for financial support and the ATNF for hospitality. This research has made use of the Simbad database, operated at CDS, Strasbourg, France. All FFT computing in the reduction proces was performed on the Cray-C98 of the National Facility for Supercomputing in Amsterdam.

\section{References}

Bowers P.F., Knapp G.R., 1989, ApJ 347, 325 (03)

Braz A., Epchtein N., 1983, A\&AS 54, 167 (02)

Habing H.J., 1996, ARA\&A 7, 97

Sault R.J., 1994, A\&AS 107, 55

Sault R.J., Teuben P.J., Wright M.C.H., Astronomical Data Analysis Software and Systems IV, p. 433

Sevenster M.N., Chapman J.M., Habing H.J., Killeen N.E.B, Lindqvist M., 1996, A\&AS 122, 79 (Paper I)

te Lintel Hekkert P., Versteege-Hansel H.A., Habing H.J., Wiertz M., 1989, A\&AS 78, 399 (01)

te Lintel Hekkert P., Caswell J.L., Habing H.J., Haynes R.F., Norris R.P., 1991, A\&AS 90, 327 (PTL) (04)

van der Veen W., Habing H.J., 1988, A\&A 194, 125

Wieringa M., Kesteven M., 1992, AT Tech. Doc. Ser. 39.3, 024

Zijlstra A.A., te Lintel Hekkert P., Pottasch S.R., et al., 1989, A\&A 217, 157 
Table 1. Compact $\mathrm{OH}-$ maser sources in the galactic Disk region

The columns of Table 1 contain the following information:

1. Sequence number (coincident with spectra in Fig. 6)

2. Name in the $\mathrm{OH} \ell-b$ convention

3. Type indication

$* \mathrm{D}=$ double-peaked spectrum

$* \mathrm{~S}=$ single-peaked spectrum

$* \mathrm{I}=$ irregular spectrum.

4. Right ascension of the brightest peak for epoch J2000 (typical error $0 . ' 25)$

5. Declination of the brightest peak for epoch J2000 (typical error 0. . 5 )

6. Mean scatter of the position of the star in all channels where it was detected, in arcseconds on the sky (Sect. 5.2)

7. Radial offset of the source from pointing centre

8. Line-of-sight velocity with respect to the LSR of the blueshifted (L) peak. For S and I spectra the velocity of the peak is always given as blue-shifted for reasons of tabulation (typical error $1 \mathrm{~km} \mathrm{~s}^{-1}$ ).

9. Same for the red-shifted $(\mathrm{H})$ peak (typical error $1 \mathrm{~km} \mathrm{~s}^{-1}$ ).

10. Stellar velocity (typical error $\left.1 \mathrm{~km} \mathrm{~s}^{-1}\right)$. $v_{\mathrm{c}}=0.5 \cdot\left(v_{\mathrm{H}}+v_{\mathrm{L}}\right)$.

11. Outflow velocity; zero for $\mathrm{S}$ and I sources (typical error $\left.1 \mathrm{~km} \mathrm{~s}^{-1}\right)$.

$$
v_{\exp }=0.5 \cdot\left(v_{\mathrm{H}}-v_{\mathrm{L}}\right) .
$$

12. Flux density, corrected for primary-beam attenuation, of the blue-shifted (L) peak (typical error 5\%).

13. Same for the red-shifted $(\mathrm{H})$ peak (typical error $5 \%$ ).

14. Empirical noise in all empty planes for the present field (Sect. 5.1, Fig. 1)

15. Reference for previous $\mathrm{OH}$-maser detection (see Table 2 and Sect. 5.6)

16. Nearest IRAS PSC position (Sect. 5.5)

17. Ratio between the size of the error ellipse of the nearest IRAS point source and the distance to nearest IRAS PSC position in the direction of the $\mathrm{OH}$ position (Sect. 5.5)
Table 2. References for previous $\mathrm{OH}$ maser detections (Table 1, Col. 15)

- 01 te Lintel Hekkert et al. (1989)

- 02 Braz \& Epchtein (1983)

- 03 Bowers \& Knapp (1989)

- 04 te Lintel Hekkert et al. (1991). 